\title{
Landau levels from the Bethe Ansatz equations
}

\author{
K. Hoshi \\ Department of Applied Physics, University of Tokyo, 7-3-1 Hongo Bunkyo-ku, Tokyo 113-8656, Japan \\ Y. Hatsugai* \\ Department of Applied Physics, University of Tokyo, 7-3-1 Hongo Bunkyo-ku, Tokyo 113-8656, Japan \\ PRESTO, JST, Japan
}

(Received 18 October 1999)

\begin{abstract}
The Bethe ansatz (BA) equations for the two-dimensional Bloch electrons in a uniform magnetic field are treated in the weak-field limit. We have calculated energies near the lower boundary of the energy spectrum up to the first nontrivial order. It corresponds to calculating a finite size correction for the excitation energies of the BA solvable lattice models and gives the Landau levels in the present problem.
\end{abstract}

The system of two-dimensional Bloch electrons in a magnetic field has very rich structure. The ratio of a lattice constant and a magnetic length, which are two fundamental length scales, is crucially important. The incommensurability brings fractal structures in the problem, which can be observed in the energy spectrum and the wave functions. ${ }^{1} \mathrm{Fa}-$ mous Hofstadter's butterfly diagram is a typical demonstration of the structure. It is fractal and has a self-similar structure. It also has an interesting relation to the quasiperiodic systems (quasicrystals). ${ }^{2}$

Recently intrinsic importance of a quantum-mechanical phase is stressed as a geometrical phase, which is essential in the Aharonov-Bohm effect, the Berry's phase, anyons and the quantum Hall effect. ${ }^{3}$ The Bloch electrons in a magnetic field also have importance as a stage of a typical realization of the geometrical phase. It was first noticed by Zak in the study of a magnetic translation group. ${ }^{4}$ The effect of the geometrical phase for the Bloch electrons was pursued in the studies of the quantum Hall effect and fundamental relations between the Hall conductance and several topological invariants were discovered (the Chern number ${ }^{5}$ and the winding number on the complex energy surface ${ }^{6}$ ). This is a geometrical aspect of the problem.

For an algebraic property of the problem that originates from the magnetic translation group, there was a breakthrough recently. Wiegmann and Zabrodin found a relation between the Hamiltonian of the Bloch electrons in a magnetic field and the quantum group $U_{q}\left(s l_{2}\right){ }^{7,8}$ Using the relation, the Schrödinger equation is rewritten as a functional equation and the Bethe ansatz (BA) equations are derived. The quantum group is a relatively new mathematical concept. It is a kind of generalization of the Lie algebra that is characterized by the so-called $q$ parameter. This $q$ parameter represents the noncommutativity of the elements in the quantum group that is related to the noncommutativity of the magnetic translation group. Mathematically rich structures of the quantum group enable us to obtain insights of the physical problem. Since the BA equations are high degree multivariable ones, it is difficult to obtain an explicit solution. It usually occurs for the BA equations of the BA solvable lattice models (the $X X Z$ chain and the Hubbard chain). However, for the present problem, the explicit solution of the BA equations for the zero energy was obtained by Hatsugai-Kohmoto-Wu. ${ }^{9,10}$ When the flux per plaquette is irrational, the distribution function of the BA roots is nowhere differentiable and the polynomial representation of the $U_{q}\left(s l_{2}\right)$ gives a polynomial with quasiperiodic coefficients. It implies that the wave function has a multifractal character. Although this explicit solution is only restricted to the zero energy, the other energies were also studied numerically. ${ }^{10}$ Abanov, Talstra and Wiegmann gave beautiful results based on the string type ansatz when the flux per plaquette is an irrational golden mean. ${ }^{11}$ Krasovsky derived an integral equation for the distribution function of the BA roots in the weak-field limit and calculated the energy of the lowest energy band. ${ }^{12}$ This corresponds to calculating a finite size correction of the ground-state energy in the usual BA solvable models.

In this letter, we focus on the weak-field limit $\phi \rightarrow 0$ where the flux per plaquette is given by $\phi=B a^{2} / \Phi_{0}$ in units of a flux quantum $\Phi_{0}=h c / e$ ( $a$ is a lattice constant and $B$ is a magnetic field). Physically, this weak-field limit can be understood as a continuum limit from the lattice system where the lattice spacing $a$ vanishes with the magnetic field $B$ fixed. We calculate energies of the lowest several states analytically up to the lowest nontrivial order in $\phi$. It corresponds to calculating finite size corrections for excited state energies of the usual BA solvable lattice models. Mathematically, this weak-field limit is a semiclassical limit where the classical $s l_{2}$ is recovered from the quantum $U_{q}\left(s l_{2}\right)$.

The Hamiltonian of electrons on a square lattice in a uniform magnetic field is given by

$$
H=T_{x}+T_{y}+\text { h.c. }
$$

where $T_{x}$ and $T_{y}$ are the covariant translation operators $T_{x}$ $=\Sigma_{m, n} c_{m, n}^{\dagger} e^{i \theta_{m, n}^{x}} c_{m+1, n}, \quad T_{y}=\Sigma_{m, n} c_{m, n}^{\dagger} e^{i \theta_{m, n}^{y}} c_{m, n+1}, \quad$ and $c_{m, n}$ is an annihilation operator for an electron at a site $(m, n)$. Discrete rotation of the phases $\theta_{m, n}^{x}$ and $\theta_{m, n}^{y}$ gives a flux per plaquette $\phi: \sum_{\text {plaquette }} \theta_{m, n}=\theta_{m, n}^{x}+\theta_{m+1, n}^{y}-\theta_{m, n+1}^{x}$ $-\theta_{m, n}^{y}=2 \pi \phi$. We assume the flux $\phi=P / Q$ with mutually prime integers $P$ and $Q$. In the diagonal gauge $\left[\theta_{m, n}^{x}\right.$ $\left.=-\pi(m+n) \phi, \theta_{m, n}^{y}=\pi(m+n+1) \phi\right]$, the Hamiltonian in the momentum space is given as $H=\Sigma_{\mathbf{k}} H(\mathbf{k})$, where $H(\mathbf{k})$ 
(a)

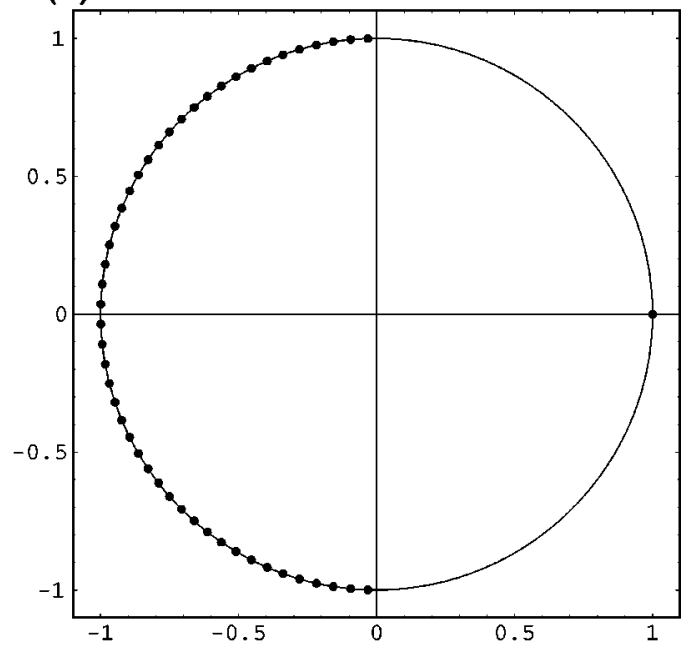

(b)

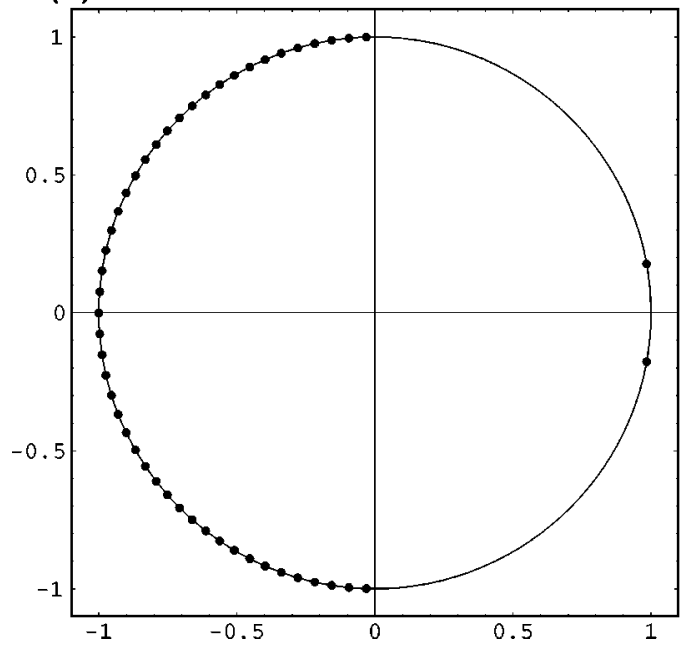

FIG. 1. Numerical solutions for the roots of the Bethe ansatz equations: (a) for the second lowest energy band and (b) for the third band $(\phi=1 / 50)$.

$=\left(e^{-i k_{x}} X+e^{i k_{y}} X^{-1}\right) Y+Y^{-1}\left(e^{-i k_{y}} X+e^{i k_{x}} X^{-1}\right) \quad$ with $2 Q$ $\times 2 Q$ matrices $X$ and $Y\left[X_{i j}=1\right.$ if $i-j \equiv 1(\bmod 2 Q), X_{i j}$ $=0$ otherwise, $\left.Y=\operatorname{diag}\left(q, q^{2}, \ldots, q^{2 Q}\right)\right]$. When one takes $P$ to be odd and chooses momenta $\left(k_{x}, k_{y}\right)$ on the so-called midband line $\left[k_{+}=\left(k_{x}+k_{y}\right) / 2 \equiv \pi / 2(\bmod \pi / Q)\right]$, the Hamiltonian is given by

$$
H_{\text {midband }}=i\left(q-q^{-1}\right)\left\{\rho_{c}(B)+\rho_{c}(C)\right\},
$$

where $\rho_{c}(B)$ and $\rho_{c}(C)$ supplemented by $\rho_{c}(A), \rho_{c}(D)$ are the cyclic representations of the quantum group $U_{q}\left(s l_{2}\right)$ $=\{A, B, C, D\} .{ }^{7,10}$ Here, the quantum group enters in the problem where the $q$ parameter is given by $q=e^{i \pi \phi}$. Since we consider the case $\phi=P / Q$, it implies that the $q$ is a root of unity $\left(q^{2 Q}=1\right)$. This cyclic representation is only possible for the $q$ that is a root of unity. The classical $s l_{2}$ is a spin algebra and it has a usual highest weight representation given by the differential operators. Correspondingly, the quantum group $U_{q}\left(s l_{2}\right)$ also has a highest weight representation given by the difference operators. The highest weight representation implies that finite degree polynomials can span the bases. On the other hand, the cyclic representation does not have a classical correspondence. However, using a relation between the highest weight representation and the cyclic representation, the discrete Schrödinger equations of the Bloch electron in a magnetic field are extended to a functional equation for a polynomial $\Psi(z)=\prod_{k=1}^{Q-1}\left(z-z_{k}\right)$ of the degree $Q-1$ (Ref. 7) as

$$
i\left(z^{-1}+q z\right) \Psi(q z)-i\left(z^{-1}+q^{-1} z\right) \Psi\left(q^{-1} z\right)=\varepsilon \Psi(z) .
$$

Putting $z=z_{m}$ and also comparing the coefficients of the highest order term, we have the BA type equations ${ }^{7}$ as

$$
\begin{gathered}
\frac{z_{m}^{2}+q}{q z_{m}^{2}+1}=q^{Q} \prod_{k=1}^{Q-1} \frac{q z_{m}-z_{k}}{z_{m}-q z_{k}}, \quad m=1,2, \ldots, Q-1, \\
\varepsilon=i q^{Q}\left(q-q^{-1}\right) \sum_{k=1}^{Q-1} z_{k} .
\end{gathered}
$$

For the semiclassical case $\phi=1 / Q$, which we consider in the paper, all the BA roots are on a unit circle, $\left|z_{k}\right|=1$ ( $k$ $=1,2, \ldots, Q-1)$ (Ref. 10) (see Fig. 1). Therefore, we set $z_{k}=e^{i \varphi_{k}}\left(\varphi_{k} \in \mathbf{R}\right)$. Then the BA Eq. (2) is written as

$$
\frac{\cos \left(\varphi_{m}-\frac{\pi}{2 Q}\right)}{\cos \left(\varphi_{m}+\frac{\pi}{2 Q}\right)}=-\prod_{k=1}^{Q-1} \frac{\sin \frac{1}{2}\left(\varphi_{m}-\varphi_{k}+\frac{\pi}{Q}\right)}{\sin \frac{1}{2}\left(\varphi_{m}-\varphi_{k}-\frac{\pi}{Q}\right)}
$$

In the limit $Q \rightarrow \infty$, the distribution function $\rho(\varphi)$ of the BA roots is well defined and the energy is given by

$$
\varepsilon=2 \pi \int_{-\pi}^{\pi} e^{i \omega} \rho(\omega) d \omega
$$

where $\rho\left(\varphi_{k}\right)=\lim _{Q \rightarrow \infty} 1 / Q \delta_{k}$ and $\delta_{k}=\varphi_{k+1}-\varphi_{k}$. Recently, Krasovsky calculated the energy of the lowest band up to the $1 / Q$ order and an integral equation for $\rho$ was derived. ${ }^{12} \mathrm{Ex}-$ tending the method in Ref. 12, we calculate energies of the general bands in the vicinity of the lower boundary of the spectrum $[\varepsilon=-4+O(1 / Q)]$. To obtain the energies, one needs the distribution of the BA roots. For the $n$-th lowest energy band with $n=O(1), Q-n$ roots are almost uniformly distributed on the left semicircle with a unit radius and the other $n-1$ roots are on the right semicircle ${ }^{10}$ (see Fig. 1). We determine the arguments of the $n-1$ roots up to the order of unity. We denote the arguments of the $Q-n$ roots and those of the other $n-1$ roots as $\theta$ and $\tau$, respectively $[\theta \in(\pi / 2,3 \pi / 2)$ and $\tau \in(-\pi / 2, \pi / 2)]$. In general, the $n-1$ roots consist of clusters separated by finite distances. The roots within each cluster converge to the single point in the $Q \rightarrow \infty$ limit. Then the arguments of the BA roots are written as $\left\{\tau_{11}, \ldots, \tau_{1 n_{1}}\right\},\left\{\tau_{21}, \ldots, \tau_{2 n_{2}}\right\}, \ldots$ where $\tau_{1 i}$ $\rightarrow c_{1}, \tau_{2 j} \rightarrow c_{2}, \ldots(Q \rightarrow \infty)\left(c_{i} \neq c_{j}, i \neq j\right)$. Further we assume $c_{i} \neq \pm \pi / 2$ (see Fig. 1). Then the BA Eq. (3) for $\tau_{11}$ in the limit $Q \rightarrow \infty$ gives 


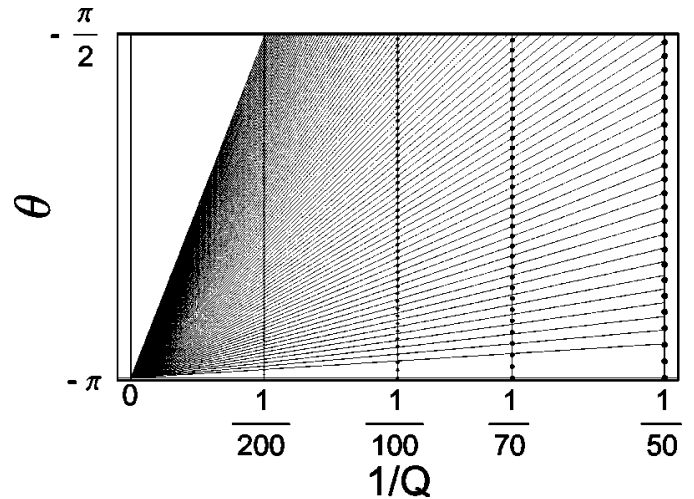

FIG. 2. Numerical solutions for the arguments of the roots $\theta_{k}$ for the third lowest band $(Q=50,70,100$, and 200). The straight lines are the asymptotic behavior for $Q \rightarrow \infty, \theta_{k}=-\pi+(k$ $+3 / 2) \pi / Q[k=1,2, \ldots,(Q-4) / 2]$.

$$
\begin{aligned}
0=\lim _{Q \rightarrow \infty} \sum_{k} \ln \left|\frac{\sin \frac{1}{2}\left(c_{1}-\theta_{k}+\frac{\pi}{Q}\right)}{\sin \frac{1}{2}\left(c_{1}-\theta_{k}-\frac{\pi}{Q}\right)}\right| \\
+\lim _{Q \rightarrow \infty} \sum_{i=2}^{n_{1}} \ln \left|\frac{\sin \frac{1}{2}\left(\tau_{11}-\tau_{1 i}+\frac{\pi}{Q}\right)}{\sin \frac{1}{2}\left(\tau_{11}-\tau_{1 i}-\frac{\pi}{Q}\right)}\right| .
\end{aligned}
$$

The first term is estimated as $\ln \left(1-\sin c_{1}\right) /\left(1+\sin c_{1}\right)$. Adding the BA equations for the roots $\left\{\tau_{11}, \ldots, \tau_{1 n_{1}}\right\}$, we have $n_{1} \ln \left(1-\sin c_{1}\right) /\left(1+\sin c_{1}\right)=0$. It means $c_{1}=0$. Similarly, we have $c_{2}=\cdots=0$. Therefore, there is only one cluster on the right semicircle (see Fig. 1) and

$$
\tau_{i} \rightarrow 0 \quad(Q \rightarrow \infty) .
$$

Now, let us calculate the rest of the BA roots $\theta_{i}$ and the energy for the $(2 p+1)$ th lowest energy band (we assume that the $Q$ is even for definiteness). By the symmetry of the BA roots distribution ${ }^{9,10,12}$ (see Fig. 1), we write arguments of the BA roots as $\theta_{0}=-\pi, \theta_{k}=-\pi+\pi k / Q+\sum_{j=0}^{k-1} \Delta_{j}$, $\theta_{-k}=-\theta_{k}, \quad k=1,2, \ldots, Q / 2-p-1 \quad$ where $\Delta_{j}=\theta_{j+1}-\theta_{j}$ $-\pi / Q$. Note that $\sum_{j=0}^{k-1} \Delta_{j}$ vanishes in the $Q \rightarrow \infty$ limit, since $\Delta_{j}=o(1 / Q)$. Moreover, $\Delta_{k}$ is exponentially small for the $k$ of the order $Q(k \sim Q)$. ${ }^{13}$ Therefore, we set $\theta_{k}=-\pi$ $+\pi k / Q+s / Q^{\delta}+o\left(1 / Q^{\delta}\right)$ for $k \sim Q$ where $s$ and $\delta$ are constants independent of $k$ and $Q$. Numerical solutions for $\theta_{k}$ are shown in Fig. 2. For the energy $\varepsilon$, we have $\varepsilon=-4$ $+4 \pi p / Q+4 s / Q^{\delta}+o\left(1 / Q, 1 / Q^{\delta}\right)$ from Eq. (2). To fix the energy, we calculate $s$ and $\delta$. Now let us rewrite the BA Eq. (3) by $\theta_{k}$ and $\tau_{l}$ as

$$
\begin{aligned}
\ln \left|\frac{\cos \left(\theta_{m}-\frac{\pi}{2 Q}\right)}{\cos \left(\theta_{m}+\frac{\pi}{2 Q}\right)}\right|=\sum_{k} \ln \left|\frac{\sin \frac{1}{2}\left(\theta_{m}-\theta_{k}+\frac{\pi}{Q}\right)}{\sin \frac{1}{2}\left(\theta_{m}-\theta_{k}-\frac{\pi}{Q}\right)}\right| \\
+\sum_{l} \ln \left|\frac{\sin \frac{1}{2}\left(\theta_{m}-\tau_{l}+\frac{\pi}{Q}\right)}{\sin \frac{1}{2}\left(\theta_{m}-\tau_{l}-\frac{\pi}{Q}\right)}\right| .
\end{aligned}
$$

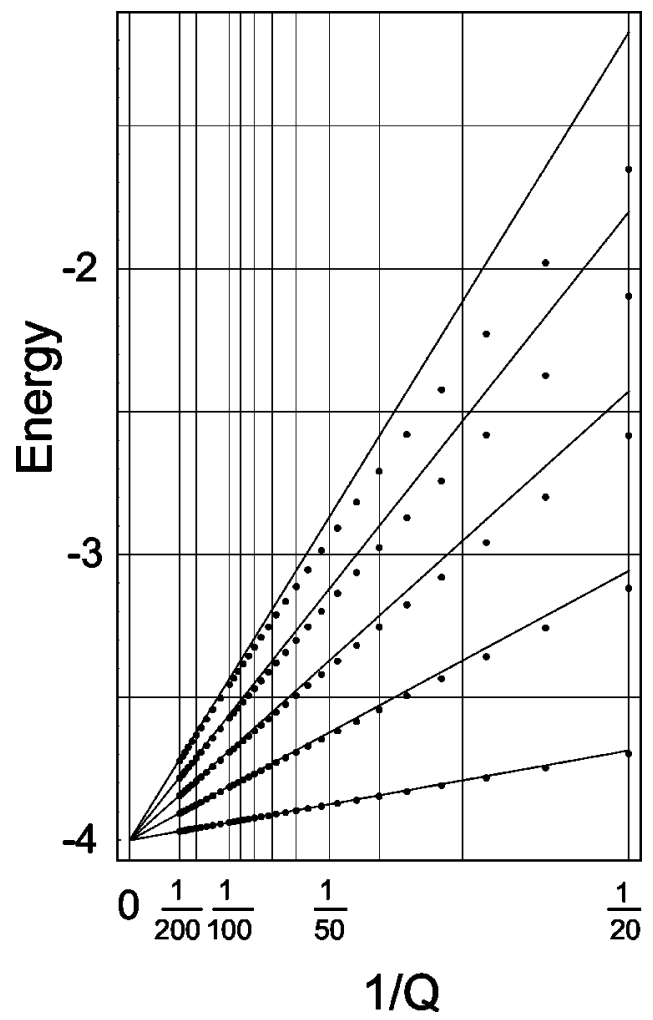

FIG. 3. Numerical results for the energies of several bands near the lower boundary. Asymptotic behaviors are shown by the solid lines.

Note that if the first summation is naively estimated as integral, the integrand diverges at the points $|m-k| \ll Q[x \ll y$ means $x / y \rightarrow 0(Q \rightarrow \infty)]$. In order to avoid this, we divide the summation into two parts $S$ and $R$, where $S$ is the contribution from $k=m-N, \ldots, m+N(1 \ll N \ll Q)$ and $R$ is that from the rest $k$ [we take $m$ such that $\lim Q \rightarrow \infty \theta_{m} \neq$ $\pm \pi / 2]$. After canceling numerators and denominators in suitable pairs, $S$ is estimated as $-\ln (1+\sin \pi m / Q) /(1$ $-\sin \pi m / Q)+r_{m} / Q^{\beta_{m}}+o\left(1 / Q^{\beta_{m}}\right)$ where $Q^{2 / 3} \ll N \ll Q$ and $r_{m}$ and $\beta_{m}$ are constants independent of $Q$. Since we have two parameters $s / Q^{\delta}$ and $r_{m} / Q^{\beta_{m}}$, we need another set of (similar) equations for the BA roots, which can be derived from the functional Eq. (1). ${ }^{12}$ From the two sets of the equations, we can fix the parameters as $\delta=\beta_{m}=1, \quad s=\pi\left(p+\frac{1}{2}\right)$. Therefore, the asymptotic distribution of the $\theta_{k}$ is determined as (see Fig. 2)

$$
\theta_{k}=-\pi+\left(k+p+\frac{1}{2}\right) \frac{\pi}{Q}+o\left(\frac{1}{Q}\right)(k \sim Q) .
$$

Cases for the $2 p$ th bands are also discussed in the similar way. To summarize the results, the energy of the $(n+1)$ th lowest band $[n=0,1,2, \ldots ; n=O(1)]$ is given as

$$
\varepsilon_{n}=-4+\left(n+\frac{1}{2}\right) \frac{4 \pi}{Q}+o\left(\frac{1}{Q}\right) .
$$

In Fig. 3, the five lowest energies are plotted as a function of $1 / Q(20 \leqslant Q \leqslant 200)$ and the straight lines are the asymptotic behavior in Eq. (5). We can also treat the general energy bands near the higher boundary of the spectrum. These ( $n$ 
+1)th highest energies are given by $\varepsilon_{n}^{\prime}=4-\left(n+\frac{1}{2}\right) 4 \pi / Q$

$+o(1 / Q)$ by the parallel argument.

Now, let us discuss the physical outcome of the results. In the absence of the magnetic field, one can recover the parabolic dispersion in the continuum model from the tightbinding model as

$E_{k}=-2 t\left(\cos k_{x} a+\cos k_{y} a\right) \stackrel{a \rightarrow 0}{\rightarrow}-4 t+t a^{2} k^{2}=-4 t+\frac{\hbar^{2} k^{2}}{2 m^{*}}$.

It implies that the effective mass is given by $m^{*}=\hbar^{2} / 2 a^{2} t$. Since $1 / Q=\phi=B a^{2} / \Phi_{0}=e B a^{2} / h c$, we rewrite the above result (5) with this effective mass $m^{*}$ as

$$
\begin{gathered}
E_{n}=t \varepsilon_{n} \rightarrow-\frac{2 \hbar^{2}}{m^{*}} \frac{1}{a^{2}}+\hbar \omega_{c}\left(n+\frac{1}{2}\right)(a \rightarrow 0), \\
n=0,1,2, \ldots ; n=O(1),
\end{gathered}
$$

where $\omega_{c}=e B / m^{*} c$. These are usual energies of the Landau levels except the diverging energy shift. Now, we can analytically recover the Landau levels from the BA equations of the Bloch electrons in a magnetic field. ${ }^{14}$

We thank Y. Morita for fruitful discussions. Y.H. was supported in part by a Grant-in-Aid from the Ministry of Education, Science, and Culture of Japan. The computation in this work has been partly done at the YITP Computing Facility and at the Supercomputer Center, ISSP, University of Tokyo.
*Electronic address: hatsugai@coral.t.u-tokyo.ac.jp

${ }^{1}$ D.R. Hofstadter, Phys. Rev. B 14, 2239 (1976).

${ }^{2}$ H. Hiramoto and M. Kohmoto, Int. J. Mod. Phys. B 6, 281 (1992).

${ }^{3}$ Geometric Phases in Physics, edited by A. Shapere and F. Wilczek (World Scientific, Singapore, 1989).

${ }^{4}$ J. Zak, Phys. Rev. 134, 1602 (1964).

${ }^{5}$ D.J. Thouless, M. Kohmoto, P. Nightingale, and M. den Nijs, Phys. Rev. Lett. 49, 405 (1982).

${ }^{6}$ Y. Hatsugai, Phys. Rev. B 48, 11851 (1993); Phys. Rev. Lett. 71, 3697 (1993).

${ }^{7}$ P.B. Wiegmann and A.V. Zabrodin, Nucl. Phys. B 422, 495 (1994); Mod. Phys. Lett. B 8, 311 (1994); Phys. Rev. Lett. 72, 1890 (1994).

${ }^{8}$ L.D. Faddeev and R.M. Kashaev, Commun. Math. Phys. 169, 181 (1995).

${ }^{9}$ Y. Hatsugai, M. Kohmoto, and Y.S. Wu, Phys. Rev. Lett. 73, 1134 (1994).

${ }^{10}$ Y. Hatsugai, M. Kohmoto, and Y.S. Wu, Phys. Rev. B 53, 9697
(1996).

${ }^{11}$ A.G. Abanov, J.C. Talstra, and P.B. Wiegmann, Phys. Rev. Lett. 81, 2112 (1998); Nucl. Phys. B 525, 571 (1998); P.B. Wiegmann, Prog. Theor. Phys. Suppl. 134, 171 (1999).

${ }^{12}$ I.V. Krasovsky, Phys. Rev. B 59, 322 (1999).

${ }^{13}$ For the $k$ of the order $Q$, one can estimate $\Delta_{k}$ as $\Delta_{k}$ $=\left(\Delta_{k} / \Delta_{k-1}\right) \cdots\left(\Delta_{k / 2+1} / \Delta_{k / 2}\right) \Delta_{k / 2} \leqslant\left(\Delta_{k / 2+1} / \Delta_{k / 2}\right)^{k / 2} \Delta_{k / 2}$ $\leqslant C \chi\left(\theta^{*}\right)^{-\alpha Q}$ where $\theta^{*}$ and $\alpha$ are constants independent of $Q$ such that $\chi\left(\theta^{*}\right)<1$ and $0<\alpha<1$ (Ref. 12). Therefore, $\Delta_{k}$ is exponentially small for the $k$ of the order $Q$.

${ }^{14}$ To show the Landau degeneracy, we have to show that each of the bandwidth vanishes in the semiclassical limit even after the rescaling $\varepsilon_{n} \rightarrow E_{n}=t \varepsilon_{n}=\hbar^{2} / 2 m^{*} 1 / a^{2} \varepsilon_{n}(a \rightarrow 0)$. However, the sum of the $Q$ energy bandwidths calculated numerically scales as $\sim C t / Q$ (Ref. 15). It implies that each bandwidth scales as $\sim C t / Q^{2}=C\left(\hbar^{2} / 2 m * a^{2}\right)\left(e B a^{2} / h c\right)^{2} \sim C^{\prime} a^{2}$. If we assume it, the bandwidth vanishes in the continuum limit $(a \rightarrow 0)$. To show the degeneracy analytically is an interesting future problem.

${ }^{15}$ D.J. Thouless, Phys. Rev. B 28, 4272 (1983). 